\title{
Mapas Conceptuales y la Instrucción Matemática
}

RESPONSABLE: $M g r$ Rolando Wilman Vásquez Jaico

\begin{abstract}
RESUMEN. Los mapas conceptuales son una nueva técnica de enseñanza y de aprendizaje que estimula al alumno a aprender a aprender, al mismo tiempo que es una herramienta poderosa en manos del profesor de Matemática. Es decir, el objetivo que se pretende es que el alumno disponga de una herramienta eficaz para que, por si mismo, pueda generar nuevos conocimientos ante la presentación de nuevas situaciones o informaciones. Desde el punto de vista del profesor, este contará con un medio que le permitirá recoger el sentir de la clase, asi como con distintas posibilidades para insertarlas en el proceso de enseñanza de la Matemática.
\end{abstract}

Palabras claves: mapa conceptual, palabra enlace, proposición, jerarquización, selección.

\author{
Miembros: $\operatorname{Mgr}$ CPC. Betty Cohaila Calderón
}

Ing. Econ. Juan Cáceres Mamani

\begin{abstract}
Conceptual maps are a new technique of teaching and learning which encourages student learning to learn at the same time is a powerful tool in the hands of the mathematics teacher. Is say, the aim is that the student has a effective tool for that, by itself, can generate new knowledge before the presentation of new situations or information. From the point of view the teacher, it will be a means of allowing you to collect the feel the class, as well as ways to insert it in the teaching of the mathematical process.
\end{abstract}

Keywords: Conceptual map, Word link, proposition, hierarchization, selection.
INTRODUCCIÓN. A pesar de que la robustez y eficacia de los mapas conceptuales han sido contrastadas mediante una abundante validación empírica en la última década, creemos necesario exponer una variedad amplia de experiencias de la utilización de esta técnica, en distintos niveles de la enseñanza, para aprender de la experiencia de los profesores que la han aplicado.

Los mapas conceptuales constituyen un método para mostrar, tanto al profesor como al alumno, el organigrama jerárquico conceptual que sobre un tema determinado o disciplina posee el alumno. La organización y el grado de diferenciación de conceptos de la estructura cognitiva del alumno se hace, de esta forma, patente y manifiesta.

Desde la perspectiva de la Matemática es importante que los estudiantes logren la comprensión de los diversos objetos matemáticos y que transfieran los conocimientos previos de modo que sean capaces de tomar buenas decisiones al momento de identificar los modelos matemáticos que cada persona necesita en su situación particular.

Es muy importante el análisis cualitativo de un proceso de estudio matemático. Estos procesos presentan gran complejidad, por la variedad de objetos y relaciones que es necesario tener en cuenta en las dimensiones epistémica, cognitiva e instruccional involucradas. La valoración de la idoneidad de tales procesos requiere observar, registrar y medir (usando diversos métodos y técnicas) un complejo de informaciones sobre el estado y evolución de los distintos componentes y dimensiones que lo definen. La descripción de este complejo de datos analizados se realiza con base en un modelo teórico de la cognición y de la instrucción matemática. En este trabajo, nos apoyamos en la Teoría de las Funciones Semióticas (TFS) (Godino, 2003) para analizar una secuencia de un proceso de estudio matemático.

\section{MATERIAL Y MÉTODOS}

Análisis cualitativo

Para la realización de esta investigación se aplicó el concepto de observable, definido como aquella entidad (objeto o hecho) que puede ser identificada por un observador que toma por referencia una determinada teoría. La estructuración jerarquizada de observables constituye una red objetiva, que formaliza la noción de mapa conceptual dentro de dicha teoría en el siguiente modo: la validación o refutación de una hipótesis queda determinada por la existencia o no de una red objetiva.

También se aplicó el concepto de evolución de una teoria, definida como: la evolución de una teoria viene determinada por el contraste entre un análisis a priori y un análisis a posteriori. La teoria busca validar las hipótesis que formula (a priori). Los hechos observados permiten (a posteriori) validar o refutar, total o parcialmente, las hipótesis enunciadas. Este proceso de contraste es consustancial a la teoría subyacente; la teoria determina las entidades que podrán ser identificadas en el sistema y los instrumentos de observación y toma de datos. Cuando el observador analiza un sistema concreto, trata de identificar los elementos primarios como partes de dicho sistema y construye a partir de elios una red objetiva que modeliza según la teoría el sistema observado. 


\section{Redes objetivas}

El análisis de una clase de matemáticas sobre expresiones algebraicas a estudiantes universitarios. El ejemplo corresponde a un fragmento de una sesión de clase convencional. El objetivo de la enseñanza observada es que los estudiantes recuerden, interpreten $\mathrm{y}$ formalicen las definiciones de expresiones algebraicas aplicándolas en una situación que pone en juego conocimientos de la Matemática: factorización de una expresión algebraica. En el proceso total de la situación propuesta se utilizan dos clases de 45 minutos; la secuencia que analizamos es una parte (15' aprox.). El significado de referencia en el proceso instruccional observado es la noción formal de expresión algebraica.

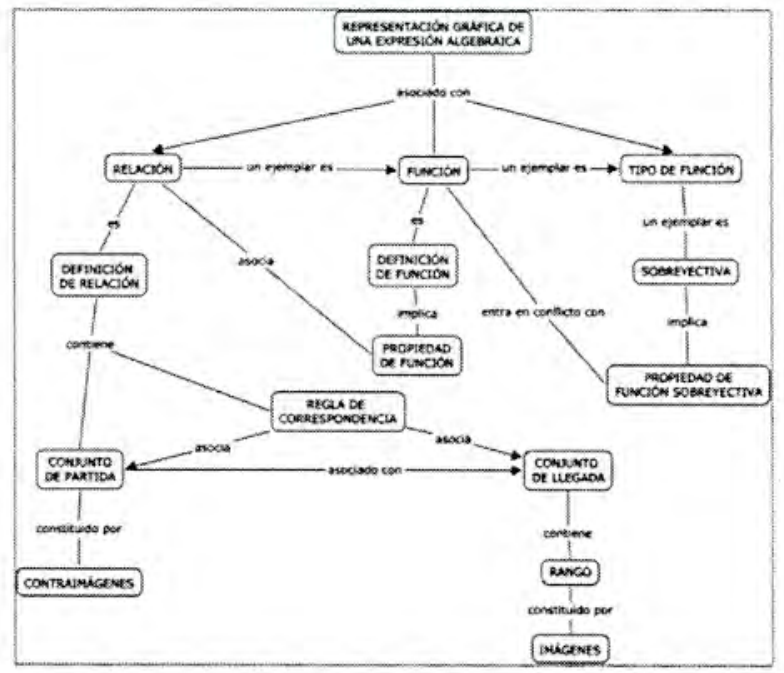

Figura 1: Vista parcial que estructura las entidades discursivas (conceptos, propiedades y argumentos).

El análisis de la Figura 1 permite identificar dos conflictos epistémicos esenciales: uno, las funciones no son aceptadas como una clase particular de correspondencia; otro, no se discriminan las características de la noción de función de las características de los "tipos de función". Además, las facetas duales de los elementos primarios se ponen de manifiesto; por ejemplo: la dualidad contenidoexpresión puede identificarse en el uso de los conceptos en los diferentes registros; la dualidad personalinstitucional, en los problemas que el profesor plantea a lo largo del proceso con la intención de hacer evolucionar el significado personal hacia el institucional pretendido; la dualidad ejemplar-tipo, en los argumentos de los estudiantes mediante ejemplos que el profesor intenta describir como representantes de una clase de objetos más general; la dualidad ostensivo-no ostensivo en el uso privilegiado del lenguaje oral que dificulta la retroalimentación del proceso de estudio, determinando la necesidad del registro escrito para facilitar el contraste de las diversas producciones de los estudiantes; la dualidad elemental-sistémico en el papel articulador que cumple la noción de función en todo el discurso, resaltando las conexiones matemáticas entre las entidades primarias involucradas.
Por tanto, se demuestra que existe relación entre los mapas conceptuales y la instrucción matemática, hipótesis de la investigación.

\section{Análisis experimental}

La metodología se aplicó a los integrantes de la Asignatura Matemática del primer año de la Facultad de Ciencias Contables y Financieras de la Universidad Nacional Jorge Basadre Grohmann de Tacna, promoción 2008-2012. El grupo experimental estuvo integrado por 54 estudiantes y se desarrolló la experiencia en 3 momentos.

\section{Primer momento}

El inicio de la actividad fue realizado para descubrir por medio de los mapas conceptuales las ideas previas de los alumnos, con la finalidad de exteriorizar lo que estos ya saben, de forma que quede a la vista tanto de él mismo como del profesor (Novak y Gowin, 1988) y también para motivarlos y sensibilizarlos sobre el tema a tratar. Para la realización de lo anterior, este momento se subdividió en fases:

a) Previo a la inducción

- Diseñar un instructivo para la elaboración de mapas conceptuales.

- Planificar un taller sobre elaboración de mapas conceptuales.

- Diseñar un esqueleto de un mapa conceptual sobre expresiones algebraicas, tal como lo sugieren Novak y Cañas (2004).

- Realizar la conformación de los equipos de trabajo, resultando un total de 9 equipos de 6 estudiantes cada uno.

\section{b) Inducción}

Se desarrolló el taller sobre mapas conceptuales en una sesión de 2 horas, en la cual se expusieron sus bases teóricas, aplicaciones y manejo. Seguidamente, se hizo entrega a cada equipo del instructivo y del esqueleto de mapa conceptual antes mencionado. Esta fase tuvo como finalidad dar la oportunidad al estudiante de aplicar lo aprendido y al profesor de orientar con sus explicaciones.

\section{C) Búsqueda de ideas previas}

En la segunda sesión de clases, cada equipo comenzó a trabajar con el tema problema de las expresiones algebraicas. El docente seleccionó este tema por considerarlo fundamental dentro del estudio de la Matemática y le solicitó a sus alumnos construir un mapa conceptual sobre lo que ellos conocen en relación con el mismo. Una vez finalizado el trabajo cooperativo, 
los 9 equipos mostraron sus preconceptos sobre el tema y las reflexiones respectivas, para luego el profesor evaluar los mapas conceptuales y diseñar, de esta manera, las estrategias de enseñanza del segundo momento, según a ese nivel de conocimientos mostrado por ellos y con el apoyo del profesor.

Segundo momento

Se utilizó la exposición oral del profesor unida a actividades de discusión, diálogo, confrontación de ideas y autorreflexión como estrategias de enseñanzaaprendizaje que propiciaran la motivación, la crítica y la reflexión; asimismo, se realizaron lecturas críticas de material bajado de Internet, resolución de problemas numéricos y construcción de preguntas y aclaratorias. Estas actividades se realizaron en dos sesiones sucesivas.

\section{Tercer momento}

Corresponde a la consolidación de conocimientos (Díaz Barriga y Hernández, 1999), la cual se realizó en una sesión final que incluyó actividades de síntesis, evaluación del aprendizaje alcanzado y retroalimentación, a través de la elaboración de un mapa conceptual que integró los contenidos aprendidos. $\mathrm{Al}$ finalizar la experiencia se entregó una encuesta con el objeto de evaluar si las estrategias aplicadas (mapas conceptuales y trabajo cooperativo) fueron útiles en su proceso de aprendizaje.

\section{RESULTADOSYDISCUSIÓN}

Para la evaluación de los mapas conceptuales antes y después de la instrucción, se consideró seleccionar aleatoriamente una muestra de 5 equipos ( 30 estudiantes en total). Se evaluaron los mapas conceptuales haciendo uso de los siguientes criterios: proposiciones válidas, jerarquización del mapa, relaciones cruzadas y ejemplos concretos. Los resultados obtenidos se presentan en la Tabla 1, de los cuales se muestra una avance significativo en las proposiciones explicativas del tema; no obstante, en el resto de los criterios no se evidencia un avance en los mapas posteriores. De esto último es importante resaltar que entre estos criterios, la ausencia de relaciones cruzadas puede ser que haya ocurrido por (1) falta de cooperación entre los miembros del equipo, (2) rigidez de sus esquemas para realizar mapas conceptuales con otra forma que no sea la lineal, reflejando, así, aprendizajes memorísticos y poco significativos, (3) la mediación cognitiva del profesor, la cual fue insuficiente para aclarar las dudas surgidas de todos los alumnos simultáneamente durante el desarrollo de las actividades.

Novak (2004) ya habia predicho que esta estrategia sería dificultosa para el profesor, sobre todo en su ayuda a los estudiantes para construir mapas conceptuales, asi como en la evaluación.

\begin{tabular}{|c|c|c|}
\hline \multirow{2}{*}{$\begin{array}{l}\text { Criterios de Evaluación } \\
\text { Proposiciones válidas }\end{array}$} & \multicolumn{2}{|c|}{$\begin{array}{l}\text { Comparación de los mapas conceptuales } \\
\text { iniciales con los finales" }\end{array}$} \\
\hline & $\begin{array}{l}\text { Existe avance } \\
4\end{array}$ & $\begin{array}{l}\text { No hay avance } \\
1\end{array}$ \\
\hline Jerarquización del mapa & 3 & 2 \\
\hline Relaciones cruzadas & 0 & 5 \\
\hline Ejemplos & 1 & 4 \\
\hline
\end{tabular}

Cabe destacar, por otro lado, que de los 5 mapas conceptuales evaluados antes de la instrucción, 3 presentaron errores conceptuales que desaparecieron finalmente en el mapa posterior. Es por ello que las encuestas facilitadas individualmente a los alumnos reflejaron mayoritariamente que consideraron los mapas conceptuales como una herramienta útil en el proceso de aprendizaje, porque les permitió "darse cuenta" de sus errores conceptuales y de comprender las relaciones entre los distintos conceptos que la conforman.

Por tanto, se demuestra que existe relación entre los mapas conceptuales y la instrucción matemática.

Un ejemplo realizado por un equipo antes y después de la instrucción (Figura 2).

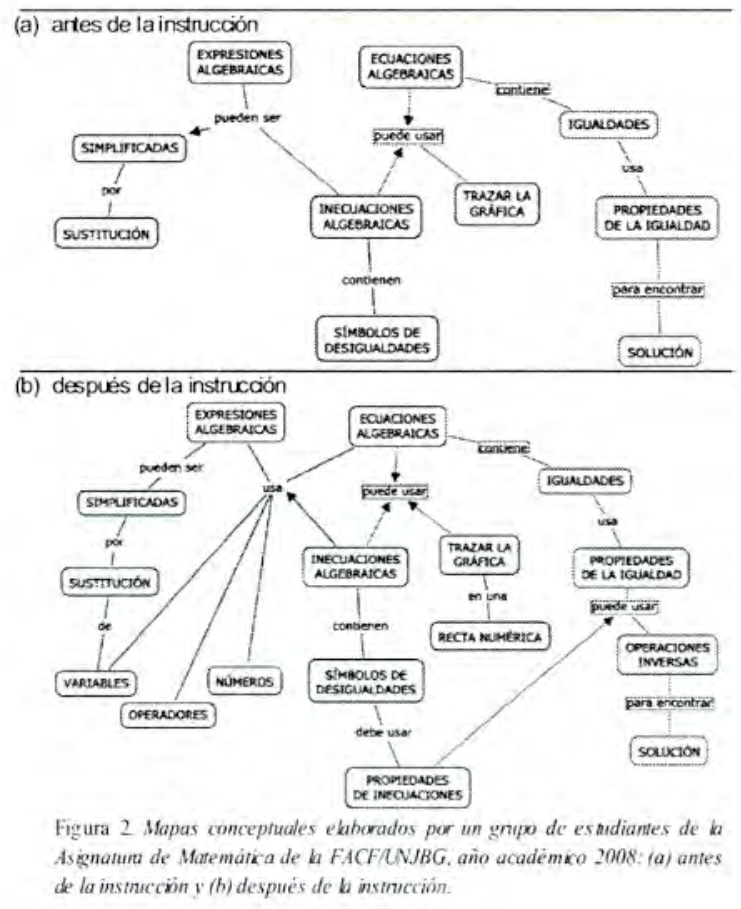

\section{CONCLUSIONES}

1. Se concluye que a pesar de que en este estudio fue dificil establecer la estrategia de mapas conceptuales en aulas, si existieron aprendizajes significativos sobre el tema problema. Esta estrategia se considera como una herramienta excelente para mejorar la comprensión de un tema de estudio y como medio para facilitar la construcción de conocimientos. 
2. De los resultados obtenidos se confirma la hipótesis de que el uso de mapas conceptuales como ayuda para el aprendizaje se muestra como un apoyo efectivo en el ámbito de análisis de modelos matemáticos y su aplicación.

\section{REFERENCIAS BIBLIOGRÁFICAS}

DÍAZ BARRIGA, F. y HERNÁNDEZ R., G. (1999). Estrategias docentes para un aprendizaje significativo. Editorial McGraw-Hill, México.

GODINO, J. D. (2003). Teoria de las funciones semióticas. Un enfoque ontológico y semiótico de la cognición e instrucción matemática. Departamento de
Didáctica de la Matemática, Universidad de Granada. [Recuperable en la dirección (21/05/2008): Http://www.ugr.es/local/jgodino].

NOVAK, J. (2004). The theory underlying concept maps and how to construct them. [Recuperable en la dirección(23/04/2008):

http://cmap.coginst.uwf.edu/info].

NOVAK, J. and CAÑAS, A. (2004). Building on New Constructivist Ideas and CmapTools to Create a New Model for Education. En A. J. Cañas, J. D.

NOVAK, J.D.; Gowin, D.B. (1988). Aprendiendo a aprender: Barcelona, Martínez Roca. 